\title{
ANTI-INFLAMMATORY EFFECT AND TOXICOLOGICAL QALIFICATION OF COMBINED PHYTOCHEMICALS ON ADJUVANT-INDUCED ARTHRITIS IN WISTAR RAT
}

\author{
MEHTAB ALAM ${ }^{\mathrm{a} 1}$, VIKAS GALAV ${ }^{\mathrm{b}}$ AND SHADAB ALAM ${ }^{\mathrm{c}}$ \\ ${ }^{a}$ Department of Taxicology, Dabar Research Foundation, Ghaziabad, U.P., India \\ ${ }^{b}$ Department of Veterinary \& Animal Science, Rajasthan University, Rajasthan, India \\ ${ }^{c}$ Life Science Research Foundation, Faridabad, Delhi NCR, India
}

\begin{abstract}
Inflammation has been implicated in many disorders, including cancer and available therapies elicit adverse effects. Thymoquinone and Diferuloylmethane have shown potency against inflammation. Inflammatory disorder to be considered autoimmune disease which, affects the joints and is associated with swelling, stiffness and pain. The anti-inflammatory study was subjected to evaluate therapeutic potential of thymoquinone and diferuloylmethane on freund's complete adjuvant induced arthritis in rats. Arthritis was induced in rats by injecting $0.1 \mathrm{ml}$ of freund's complete adjuvant into the left hind paw of the rat intradermally for 21 days. Thymoquinone, diferuloylmethane and thymoquinone + diferuloylmethane combined and alone orally administered to male and female wistar rat at dose levels of $2.5,5.0$ and10.0 mg/kg body weight for 35 days repeatedly, post induced did not produce any sign of toxicity, mortality, pathological changes and significant blood parameters changes. The investigated result showed that the thymoquinone + diferuloylmethane $(10 \mathrm{mg} / \mathrm{kgb} . \mathrm{wt})$ significantly $(p<0.05)$ inhibited the FCA induced arthritis and showed significant anti-inflammatory activity. Therefore, thymoquinone and diferuloylmethane treatment found to possess potent anti-inflammatory activity with no toxicity and the treatment significantly inhibited the development phase of arthritis which, is further supported by its anti-inflammatory effect was comparable to that of prednisolone (5 $\mathrm{mg} / \mathrm{kgb} . \mathrm{wt})$.
\end{abstract}

KEYWORDS: Thymoquinone, Diferuloylmethane, Freund's Adjuvant Induced Arthritis, Arthritis Activity

Inflammation is a common clinical conditions and rheumatoid arthritis (RA) is a chronic inflammatory autoimmune disorder that affects about 2.1 million (Majithia and Geraci, 2007) (El-Dakhakhny, 1965) in Indians and Americans etc which, affects the joints and is associated with swelling, stiffness and pain. The drugs commonly in used for the treatment of inflammation and RA include glucocorticoids eg., cortisone, prednisolone and NSAIDS drugs (eg. Ibuprofen), disease-modifying antiinflammatory and anti-rheumatic drugs (DMAIDs and DMARDs; eg. Methotrexate (MTX) and leflunomide) and biological response modifiers (eg. Tumor necrosis factoralpha blocking agents). Such therapies are helpful controlling the symptoms of acute RA, but their effect on chronic, prolong RA are unsatisfactory. Moreover, besides their high cost, the prolonged use of many of these drugs is associated with severe adverse reactions and toxicity, including gastrointestinal disturbances and cardiovascular risk.

Thymoquinone is a member of the ranunculaceae family growing in many countries. For many centuries, Nigella sativa seeds (also called black seeds or black cumin) have been used as a food additive as well as for medicinal purposes in many countries (Jansen, 1981). This plant is one of the most extensively studied, both phytochemically and pharmacologically (El-Sayed, 1998) (Riaz et al., 1996) (Siddiqui and Sharma, 1996) (Worthen et al., 1998). Most properties of whole seeds or their extracts are mainly attributed to quinone constituents, of which thymoquinone is more abundant compound (Mahfouz et al., 1960) (Filippo D'Antuono et al., 2002). Diferuloylmethane, an anti-inflammatory agent used in traditional medicine One of these traditional medicines, curcumin, is a component of the culinary spice turmeric, which is also often used in curry powder. Its active ingredient was first isolated in 1842 by Vogel. In 1910, Milobedzka determined that the structure was diferuloylmethane, and this compound was first synthesized in 1918 by Lampe and cocrystalized with 5-lipoxygenase in 2003 by SkrzypczakJankun et al., (2000).

\section{MATERIALS AND METHODS}

Male and female albino wistar rats (rattus norvegicus) were maintained at 19 to $25^{\circ} \mathrm{c}$, relative humidity between $50 \pm 20 \%$ and a light/dark cycle of 12 $\mathrm{hr}$. The rats were provided with rat pellet feed (amrut brand, pranav agro Pune) and drinking water filtered through aquaguard water filtration system ad libitum throughout the study period. All groups of rats were acclimatized 6 days

\footnotetext{
${ }^{1}$ Corresponding author
} 
prior to the start dosing. The thymoquinone sample was purchased from Sigma Aldrich India and peanut oil from local market the dose volume maintained at $5 \mathrm{ml} / \mathrm{kg}$ body weight.

\section{Complete Freund's Adjuvant Arthritis}

After randomization male and female rats $(110 \pm$ $20 \mathrm{~g}$ body weight) were divide in to six groups (I to VI) each group consist 5 male and 5 female rats. On day one, all rats were injected into the sub plantar region of the left hind paw with $0.1 \mathrm{ml}$ of Freund's complete adjuvant. This consist of mycobacterium butyricum suspended in heavy paraffin oil by thorough grinding with motor and pestle to give a concentration of $6 \mathrm{mg} / \mathrm{ml}$. Dosing with the test and standard substance was started on the first day and continued for 21 days.

\section{Anti-inflammatory Studies}

Based on the subacute 28 days oral toxicity in rats. Group I served as arthritis control group and group II served as vehicle control group given a daily dose of normal saline and peanut oil (based on the higher dose volume). The rats of group III, IV and $\mathrm{V}$ were given thymoquinone, diferuloylmethane and thymoquinone + diferuloylmethane mixed in peanut oil via gavage at dose level $2.5,5.0$ and $10 \mathrm{mg} / \mathrm{kg}$ body weight respectively for 35 days post induced arthritis and group VI served as standard drug (prednisolone) and given $5.0 \mathrm{mg} / \mathrm{kg}$ body weight. Rats were observed for the paw swelling in the injected and contra lateral hind paws of the rats were monitored daily using liquid displacement plethysmometer (Ugo Basile, Italy). Increase in the extent of erythema and edema of the tissues shows the severity of the inflammation. The difference in severity of arthritis between the experimental groups and arthritis control group were statistically analyzed and toxicological effect and mortality throughout the study period. Body weight, food and water of individual rat were recorded weekly for each group. After 56 day treatment as well as controls animals were sacrificed and blood collected directly from jugular vein in ethylene diamine tetra acetic acid (EDTA) solution and non-oxalate tubes for the estimation of haematological and biochemical parameter respectively.

\section{Paw Edema}

Paw volumes of left hind limb were recorded and measured on day $1 \mathrm{st}, 2^{\text {nd }} 3 \mathrm{rd}, 5 \mathrm{th}, 10^{\text {th }}, 14^{\text {th }}, 18^{\text {th }}$ and $21^{\text {st }}$ using mercury column plethysmometer. The 5th day measurement is indicative of primary lesions and13th day measurement were aid in estimating secondary lesions. On the day $21 \mathrm{st}$, the secondary phase of rheumatoid arthritis becomes more evident and inflammatory changes.

\section{Arthritic Index}

All the animals were closely observed for organs like ears, nose, tail, fore paws and hind paw and arthritic index (Pearson, 1959) was calculated.

\section{Organ Body Weight Ratio}

The vital organ such as liver, kidney, brain, heart, lung, spleen, adrenal of rats and the male sex organ(testis, epididymis, prostrate and seminal vesicle) and female sex organs (ovary, uterus, cervix and vagina) were quickly removed and weigh individually. The organ to body weight ratio was calculated.

\section{Biochemical Estimation}

Different biochemical parameters like Alkaline phosphatase (ALP) marker for bone destruction, Acid Phosphatase (ACP) the lysosomal enzyme activity, Serum glutamate oxalo acetate transaminase (SGOT) and Serum glutamate pyruvate transaminase (SGPT) were estimated by using ALP,ACP, SGOT and SGPT kit in Erba Mannhein EM 200 Clinical Chemistry Analyser. Bood samples were collected by sublingual rout, centrifused and supernatant serum was collected. Different enzyme reagents were added to the serum and estimated in an auto analyser.

\section{Haematological study}

Blood collected in EDTA tube was analyzed for red blood cells (RBC) and white blood cells (WBC) counts were determined according method of Winfrobe and Landsberg whereas, haemoglobbin and differential leucocytes counts (DLC) were measured according to procedure of Kolmer et al., (1995).

\section{Statistical Analysis}

Statistical significance were presented between control and experimental values as mean $\pm \operatorname{SEM}(n=5)$. Statistical comparison of body weight changes was made using one way ANOVA (Seigel, 1996). 


\section{RESULTS}

The left hind paw injected with complete freund's adjuvant become gradually swollen and reach edits peak at $21^{\text {st }}$ day. The results obtained for the different dose of treated test substance and the standard drug prednisolone $5.0 \mathrm{mg} / \mathrm{kg}$ body weight in the complete freund's adjuvantinduced (FCA) paw edema test at specific time intervals. It was obvious that during $21^{\text {st }}$ day treatment paw edema in disease control inflamed paw is increase in time dependent manner and all administration groups significantly inhibited the development of joint swelling induced by complete Freund's adjuvant. Arthritic index and rheumatoid factor were significantly $(p<0.05)$ decreased start on 30 days in treatment with thymoquinone, diferuloylmethane and thymoquinone + diferuloylmethane at $2.5,5$ and 10.0 $\mathrm{mg} / \mathrm{kg}$, and prednisolone $5.0 \mathrm{mg} / \mathrm{kg}$ treated animal as compare to disease control treatment.

A marked increase in the activity of membrane marker enzymes (ALP, SGOT and SGPT) were observed in the serum of arthritis rats (Group V).

\section{Organ Body Weight Ratio}

The absolute body weights of treated male and female rats no significant changes were observed while, comparable to controls rats. The relative organ weights (organ to body weight ratio) of animals exposed to different dose of thymoquinone did not indicate any significant changes and value are shown in (Table 1).

Table 1: Relative Organ Body Weight of Male Rats Orally Administration Thymoquinone for 35 days

\begin{tabular}{|c|c|c|c|c|c|c|}
\hline \multicolumn{7}{|c|}{ Dose (mg/kg body weight) } \\
\hline Organ & $\begin{array}{c}\text { Arthritis } \\
\text { Control }\end{array}$ & $\begin{array}{c}\text { Vehicle } \\
\text { Control }\end{array}$ & Thymoquinone & Diferuloylmethane & $\begin{array}{c}\text { Thymoquinone }+ \\
\text { Diferuloylmethane }\end{array}$ & $\begin{array}{c}\text { Standard } \\
\text { Drug }\end{array}$ \\
\hline Liver & $3.11 \pm 0.22$ & $2.91 \pm 0.22$ & $3.04 \pm 0.21$ & $3.08 \pm 0.21$ & $3.11 \pm 0.21$ & $2.95 \pm 0.22$ \\
\hline Kidney & $0.74 \pm 0.20$ & $0.76 \pm 0.34$ & $0.76 \pm 0.03$ & $0.76 \pm 0.07$ & $0.77 \pm 0.34$ & $0.76 \pm 0.27$ \\
\hline Lungs & $0.73 \pm 0.02$ & $0.71 \pm 0.22$ & $0.72 \pm 0.02$ & $0.72 \pm 0.02$ & $0.71 \pm 0.21$ & $0.75 \pm 0.26$ \\
\hline Brain & $0.74 \pm 0.03$ & $0.86 \pm 0.19$ & $0.78 \pm 0.03$ & $0.81 \pm 0.05$ & $0.87 \pm 0.18$ & $1.48 \pm 0.40$ \\
\hline Testis & $1.12 \pm 0.68$ & $1.18 \pm 0.62$ & $1.12 \pm 0.7$ & $1.12 \pm 0.13$ & $1.18 \pm 0.62$ & $1.17 \pm 0.04$ \\
\hline Epididymis & $0.32 \pm 0.53$ & $0.37 \pm 0.43$ & $0.41 \pm 0.64$ & $0.39 \pm 0.51$ & $0.36 \pm 0.22$ & $0.35 \pm 0.64$ \\
\hline $\begin{array}{c}\text { Seminal } \\
\text { Vesicle }\end{array}$ & $0.48 \pm 0.56$ & $0.46 \pm 0.42$ & $0.52 \pm 0.61$ & $0.54 \pm 0.12$ & $0.52 \pm 0.21$ & $0.49 \pm 0.53$ \\
\hline Spleen & $0.242 \pm 0.01$ & $0.24 \pm 0.01$ & $0.22 \pm 0.03$ & $0.22 \pm 0.03$ & $0.25 \pm 0.01$ & $0.53 \pm 0.26$ \\
\hline Heart & $0.29 \pm 0.00$ & $0.33 \pm 0.02$ & $0.32 \pm 0.03$ & $0.31 \pm 0.03$ & $0.33 \pm 0.02$ & $1.44 \pm 1.42$ \\
\hline Adrenal & $0.021 \pm 0.01$ & $0.02 \pm 0.06$ & $0.02 \pm 0.02$ & $0.02 \pm .001$ & $0.02 \pm 0.05$ & $0.16 \pm 0.32$ \\
\hline
\end{tabular}

Table 1 (continue): Relative Organ Body Weight of Female Rats Orally Administration Thymoquinone for 35 days

\begin{tabular}{|c|c|c|c|c|c|c|}
\hline \multicolumn{7}{|c|}{ Dose (mg/kg body weight) } \\
\hline Organ & $\begin{array}{c}\text { Arthritis } \\
\text { Control }\end{array}$ & $\begin{array}{c}\text { Vehicle } \\
\text { Control }\end{array}$ & Thymoquinone & Diferuloylmethane & $\begin{array}{c}\text { Thymoquinone }+ \\
\text { Diferuloylmethane }\end{array}$ & $\begin{array}{c}\text { Standard } \\
\text { Drug }\end{array}$ \\
\hline Liver & $2.95 \pm 0.15$ & $2.86 \pm 0.17$ & $3.02 \pm 0.25$ & $3.02 \pm 0.23$ & $2.97 \pm 0.12$ & $2.85 \pm 0.16$ \\
\hline Kidney & $0.72 \pm 0.05$ & $0.71 \pm 0.08$ & $0.75 \pm 0.04$ & $0.73 \pm 0.06$ & $0.74 \pm 0.06$ & $0.72 \pm 0.08$ \\
\hline Lungs & $0.71 \pm 0.82$ & $0.68 \pm 0.06$ & $0.71 \pm 0.03$ & $0.71 \pm 0.07$ & $0.71 \pm 0.02$ & $0.66 \pm 0.05$ \\
\hline Brain & $0.82 \pm 0.01$ & $0.78 \pm 0.03$ & $0.77 \pm 0.02$ & $0.77 \pm 0.03$ & $0.82 \pm 0.04$ & $0.80 \pm 0.01$ \\
\hline Ovary & $0.06 \pm 0.00$ & $0.06 \pm 0.01$ & $0.05 \pm 0.01$ & $0.06 \pm 0.01$ & $0.72 \pm 0.02$ & $0.06 \pm 0.01$ \\
\hline Uterus & $0.11 \pm 0.01$ & $0.12 \pm 0.01$ & $0.11 \pm 0.02$ & $0.11 \pm 0.01$ & $0.11 \pm 0.01$ & $0.14 \pm 0.01$ \\
\hline Spleen & $0.23 \pm 0.02$ & $0.21 \pm 0.02$ & $0.23 \pm 0.01$ & $0.25 \pm 0.03$ & $0.22 \pm 0.01$ & $0.23 \pm 0.01$ \\
\hline Heart & $0.24 \pm 0.01$ & $0.27 \pm 0.01$ & $0.31 \pm 0.02$ & $0.31 \pm 0.03$ & $0.32 \pm 0.02$ & $0.31 \pm 0.02$ \\
\hline Adrenal & $0.27 \pm 0.24$ & $0.02 \pm 0.01$ & $0.01 \pm .001$ & $0.02 \pm 0.01$ & $0.02 \pm 0.00$ & $0.02 \pm 0.06$ \\
\hline
\end{tabular}




\section{Biochemical Study}

The results of serum biochemical parameters of male rats are shown (Table 2). There was no change in clinic-chemical parameters of male and female rats exposed to different dose of test substance for 28 days and the values were comparable to controls rats.

Table 2: Serum Biochemical parameter in rats treated orally with thymoquinone for 35 days

\begin{tabular}{|c|c|c|c|c|c|c|}
\hline \multirow{2}{*}{ Parameter } & \multicolumn{7}{|c|}{ Dose mg/kg body weight } \\
\cline { 2 - 7 } & $\begin{array}{c}\text { Arthritis } \\
\text { Control }\end{array}$ & $\begin{array}{c}\text { Vehicle } \\
\text { Control }\end{array}$ & Thymoquinone & Diferuloylmethane & $\begin{array}{c}\text { Thymoquinone }+ \\
\text { Diferuloylmethane }\end{array}$ & $\begin{array}{c}\text { Standard } \\
\text { Drug }\end{array}$ \\
\hline AST & $19.64 \pm 14.15$ & $16.61 \pm 14.50$ & $15.84 \pm 19.18$ & $21.24 \pm 19.66$ & $16.91 \pm 30.17$ & $17.81 \pm 29.21$ \\
\hline ALT & $67.73 \pm 10.20$ & $67.64 \pm 10.25$ & $57.62 \pm 10.50$ & $57.42 \pm 10.52$ & $62.67 \pm 11.26$ & $64.77 \pm 12.15$ \\
\hline ALP & $60.61 \pm 14.15$ & $60.60 \pm 14.51$ & $60.84 \pm 19.18$ & $61.24 \pm 19.67$ & $53.91 \pm 30.27$ & $55.41 \pm 28.26$ \\
\hline $\begin{array}{c}\text { S-Bilirubin } \\
\text { (mg \%) }\end{array}$ & $1.20 \pm 0.21$ & $1.08 \pm 0.11$ & $1.25 \pm 0.16$ & $1.38 \pm 0.11$ & $1.42 \pm 0.18$ & $1.31 \pm 0.21$ \\
\hline $\begin{array}{c}\text { S- Cholesterol } \\
\text { (mg \%) }\end{array}$ & $46.82 \pm 5.01$ & $47.01 \pm 5.42$ & $56.00 \pm 11.04$ & $54.70 \pm 10.13$ & $51.50 \pm 9.48$ & $52.51 \pm 9.57$ \\
\hline $\begin{array}{c}\text { S-Albumin } \\
\text { (g\%) }\end{array}$ & $4.16 \pm 0.19$ & $4.18 \pm 0.19$ & $4.68 \pm 0.31$ & $4.38 \pm 0.28$ & $4.26 \pm 0.18$ & $4.31 \pm 0.19$ \\
\hline S-Protein(g/dl) & $7.47 \pm 0.22$ & $7.41 \pm 0.25$ & $7.76 \pm 0.18$ & $7.46 \pm 0.11$ & $7.54 \pm 0.21$ & $7.65 \pm 0.18$ \\
\hline
\end{tabular}

\section{Haematology}

The results of haematological parameters in male and female rats exposed to different doses are shown (Table
3). There was no significance changes in $\mathrm{Hb} \mathrm{RBC}, \mathrm{WBC}$ and differential leukocyte count (DLT).

Table 3: Haematological parameters in rats treated orally with thymoquinone for $\mathbf{3 5}$ days

\begin{tabular}{|c|c|c|c|c|c|c|}
\hline \multirow{2}{*}{ Parameter } & \multicolumn{7}{|c|}{ Dose mg/kg body weight } \\
\cline { 2 - 7 } & $\begin{array}{c}\text { Arthritis } \\
\text { Control }\end{array}$ & $\begin{array}{c}\text { Vehicle } \\
\text { Control }\end{array}$ & Thymoquinone & Diferuloylmethane & $\begin{array}{c}\text { Thymoquinone }+ \\
\text { Diferuloylmethane }\end{array}$ & $\begin{array}{c}\text { Standard } \\
\text { Drug }\end{array}$ \\
\hline $\mathrm{Hb}(\mathrm{mg} / \mathrm{dl})$ & $13.68 \pm 0.27$ & $14.22 \pm 0.37$ & $15.11 \pm 0.48$ & $15.27 \pm 0.37$ & $14.79 \pm 0.26$ & $14.99 \pm 0.29$ \\
\hline $\begin{array}{c}\mathrm{RBC} \\
(\mathrm{x} 10 \mathrm{6} / \mu \mathrm{L})\end{array}$ & $8.12 \pm 0.17$ & $7.91 \pm 0.22$ & $7.18 \pm 0.08$ & $7.51 \pm 0.28$ & $6.93 \pm 0.21$ & $6.83 \pm 0.23$ \\
\hline $\mathrm{WBC}(\mathrm{mm} 3)$ & $9.08 \pm 1.48$ & $9.14 \pm 1.28$ & $9.48 \pm 2.12$ & $12.42 \pm 2.47$ & $14.88 \pm 1.71$ & $14.46 \pm 1.61$ \\
\hline $\begin{array}{c}\text { Neutrophil } \\
(\%)\end{array}$ & $41.45 \pm 3.18$ & $39.76 \pm 2.78$ & $37.62 \pm 2.12$ & $36.53 \pm 2.02$ & $36.02 \pm 1.98$ & $36.82 \pm 2.01$ \\
\hline $\begin{array}{c}\text { Leucocytes } \\
(\%)\end{array}$ & $28.01 \pm 1.22$ & $29.11 \pm 1.52$ & $24.45 \pm 1.61$ & $22.62 \pm 1.72$ & $18.82 \pm 2.78$ & $19.01 \pm 2.68$ \\
\hline Monocyte (\%) & $0.34 \pm 0.12$ & $0.55 \pm 0.55$ & $0.44 \pm 0.18$ & $0.52 \pm 0.28$ & $0.72 \pm 0.24$ & $0.74 \pm 0.23$ \\
\hline $\begin{array}{c}\text { Eosionophil } \\
(\%)\end{array}$ & $1.11 \pm 0.13$ & $0.93 \pm 0.21$ & $0.72 \pm 0.27$ & $0.98 \pm 0.07$ & $1.0 \pm 0.25$ & $1.0 \pm 0.23$ \\
\hline
\end{tabular}

\section{Histopathology}

Autopsy of treated animals after 56 days of exposure revealed no significance change in their vital organs. Microscopic examination of liver, kidney, brain, testes, and ovary of rats treated with the different doses of test substance for 28 days did not shown any significant tissue damage and were comparable with those of controls rats. While, the gross pathological examination observed slightly uterus distention in two control and one treated 
ALAM ET AL.: ANTI-INFLAMMATORY EFFECT AND TOXICOLOGICAL QALIFICATION OF COMBINED...

female rat which, spontaneous and is physiological/cycle nature and did not effect on outcome of study (Table 4).

Table 4: Histopathological Observation in tissue of rats treated treated orally with thymoquinone for 35 days

\begin{tabular}{|c|c|c|c|c|c|c|c|c|c|c|c|c|}
\hline \multirow{4}{*}{ Tissue } & \multicolumn{12}{|c|}{ Number of Lesion } \\
\hline & \multicolumn{12}{|c|}{ Dose $\mathrm{mg} / \mathrm{kg}$ body weight } \\
\hline & \multicolumn{2}{|c|}{$\begin{array}{c}0 \text { (Arthritis } \\
\text { Control) }\end{array}$} & \multicolumn{2}{|c|}{$\begin{array}{c}0 \text { (Vehicle } \\
\text { Control) }\end{array}$} & \multicolumn{2}{|c|}{ 2.5(Low) } & \multicolumn{2}{|c|}{ 5.0(Mid) } & \multicolumn{2}{|c|}{ 10.0(High) } & \multicolumn{2}{|c|}{$\begin{array}{l}\text { Standard } \\
\text { Drug }\end{array}$} \\
\hline & $\mathbf{M}$ & $\mathbf{F}$ & $\mathbf{M}$ & $\mathbf{F}$ & $\mathbf{M}$ & $\mathbf{F}$ & $\mathbf{M}$ & $\mathbf{F}$ & $\mathbf{M}$ & $\mathbf{F}$ & $\mathbf{M}$ & $\mathbf{F}$ \\
\hline Liver & NAD & NAD & NAD & NAD & NAD & NAD & NAD & NAD & NAD & NAD & NAD & NAD \\
\hline Kidney & NAD & NAD & NAD & NAD & NAD & NAD & NAD & NAD & NAD & NAD & NAD & NAD \\
\hline Lungs & NAD & NAD & NAD & NAD & NAD & NAD & NAD & NAD & NAD & NAD & NAD & NAD \\
\hline Brain & NAD & NAD & NAD & NAD & NAD & NAD & NAD & NAD & NAD & NAD & NAD & NAD \\
\hline Ovary & NAD & NAD & NAD & NAD & NAD & NAD & NAD & NAD & NAD & NAD & NAD & NAD \\
\hline Testis & NAD & NAD & NAD & NAD & NAD & NAD & NAD & NAD & NAD & NAD & NAD & NAD \\
\hline Spleen & NAD & NAD & NAD & NAD & NAD & NAD & NAD & NAD & NAD & NAD & NAD & NAD \\
\hline Intestine & NAD & NAD & NAD & NAD & NAD & NAD & NAD & NAD & NAD & NAD & NAD & NAD \\
\hline Heart & NAD & NAD & NAD & NAD & NAD & NAD & NAD & NAD & NAD & NAD & NAD & NAD \\
\hline
\end{tabular}

$\mathrm{NAD}=$ No Abnormality detected, $\mathrm{M}=$ Male, $\mathrm{F}=$ Female

\section{DISCUSSION}

Most of the investigators have reported that inhibition of adjuvant-induced arthritis in rats is one of the most suitable test procedures to screen anti-arthritic agents since, it closely resembles human arthritis. Arthritis (RA), one of the chronic inflammatory diseases, systemic inflammatory disorder affecting the synovial joints and typically producing symmetrical arthritis that leads to joint destruction. In this present study, the result demonstrated the effect of thymoquinone + diferuloylmethane and prednisolone on FCA induced arthritis model in rats, liver enzymes in plasma was markedly increased in the adjuvant induced arthritic rats and significantly reduced after treated with thymoquinone $10 \mathrm{mg} / \mathrm{kg}$, and prednisolone $5.0 \mathrm{mg} / \mathrm{kg}$ when comparable to the arthritis control and vehicle control group (Table 5 and 6). Increased white blood cell count is a common feature of inflammatory reactions, especially those induced by microbial infection. So in arthritic group an increase in total leukocyte number was found. A significant reduction $(\mathrm{p}<0.05)$ in total leukocyte number was found in case of treated thymoquinone + diferuloylmethane and standard drug $5 \mathrm{mg} / \mathrm{kg}$ body weight. In present study it was found that the administration of thymoquinone + diferuloylmethane and standard drug lead to inhibition leukocyte migration which, may have beneficial effect for joint preservation. Traditional medicine has maintained greater popularity all over developing world prompted by the increase awareness and interest in medicinal plant and the use is rapidly increase generation by generation (Daswani et al., 2006) (Ogbonnia et al., 2010). The incidence of adverse effects of these herbal remedies and sometimes life-threatening conditions has been reported among various ethnic groups (Elvin-Lewis, 2001) (Chan, 2003). The result presently conducted study revealed that daily orally administration thymoquinone + diferuloylmethane found to exhibit significant antiinflammatory and the potent anti arthritic activity by significantly $(\mathrm{p}<0.05)$ altering the pathogenesis during arthritis without exerting any side effect and did not induce any treatment related observable toxic effects, with regards to the haematological parameters, biochemical parameter and histopathological when compared to its control group of animal treated with corn oil (vehicle) only throughout the study. 
ALAM ET AL.: ANTI-INFLAMMATORY EFFECT AND TOXICOLOGICAL QALIFICATION OF COMBINED...

Table 5: Mean Percentile Paw Volume Freund's Complete Adjuvant (FCA) -Induced Arthritis Male Rats

\begin{tabular}{|c|c|c|c|c|c|c|c|}
\hline \multirow{2}{*}{ Group } & \multicolumn{7}{|c|}{ Days } \\
\cline { 2 - 8 } & $\mathbf{1}$ & $\mathbf{2}$ & $\mathbf{3}$ & $\mathbf{7}$ & $\mathbf{1 0}$ & $\mathbf{1 4}$ & $\mathbf{2 1}$ \\
\hline I & $9.5 \pm 1.5$ & $18.01 \pm 1.5$ & $33.85 \pm 3.3$ & $54.73 \pm 6.4$ & $60.33 \pm 4.3$ & $68.82 \pm 0.84$ & $86.22 \pm 1.7$ \\
\hline II & $9.81 \pm 1.1$ & $18.94 \pm 3.7$ & $33.83 \pm 3.6$ & $56.35 \pm 3.7$ & $62.61 \pm 1.8$ & $68.52 \pm 1.0$ & $86.90 \pm 1.7$ \\
\hline III & $9.83 \pm 1.21$ & $20.42 \pm 6.2$ & $34.63 \pm 3.4$ & $46.34 \pm 6.84$ & $54.38 \pm 5.6$ & $60.23 \pm 6.6$ & $84.54 \pm 2.5$ \\
\hline IV & $9.91 \pm 0.8$ & $18.34 \pm 3.4$ & $34.12 \pm 3.05$ & $56.02 \pm 2.9$ & $62.42 \pm 6.9$ & $66.11 \pm 4.3$ & $86.54 \pm 3.4$ \\
\hline V & $10.48 \pm 0.7$ & $24.8 \pm 7.8$ & $34.9 \pm 3.9$ & $57.83 \pm 1.6$ & $63.30 \pm 1.5$ & $68.11 \pm 1.5$ & $86.79 \pm 2.1$ \\
\hline VI & $4.01 \pm 0.5$ & $5.6 \pm 3.7$ & $10.11 \pm 1.0$ & $9.88 \pm 1.3$ & $61.38 \pm 5.2$ & $67.78 \pm 1.6$ & $86.38 \pm 2.5$ \\
\hline
\end{tabular}

Table 5 (continue): Mean Percentile Paw Volume Freund's Complete Adjuvant (FCA) -Induced Arthritis Female Rats

\begin{tabular}{|c|c|c|c|c|c|c|c|}
\hline \multirow{2}{*}{ Group } & \multicolumn{9}{|c|}{ Days } \\
\cline { 2 - 9 } & $\mathbf{1}$ & $\mathbf{2}$ & $\mathbf{3}$ & $\mathbf{7}$ & $\mathbf{1 0}$ & $\mathbf{1 4}$ & $\mathbf{2 1}$ \\
\hline I & $9.77 \pm 0.97$ & $19.74 \pm 3.8$ & $34.17 \pm 3.1$ & $54.70 \pm 7.3$ & $60.46 \pm 3.9$ & $69.27 \pm 1.4$ & $86.59 \pm 1.8$ \\
\hline II & $9.82 \pm 1.0$ & $18.94 \pm 3.8$ & $33.84 \pm 3.4$ & $54.68 \pm 6.6$ & $60.45 \pm 4.5$ & $68.52 \pm 1.0$ & $86.99 \pm 1.8$ \\
\hline III & $12.64 \pm 3.9$ & $29.94 \pm 5.77$ & $35.19 \pm 3.4$ & $48.75 \pm 6.5$ & $56.35 \pm 3.8$ & $64.51 \pm 2.85$ & $84.62 \pm 2.7$ \\
\hline IV & $12.14 \pm 1.4$ & $23.63 \pm 1.8$ & $33.36 \pm 1.9$ & $57.30 \pm 2.7$ & $55.50 \pm 13.41$ & $66.14 \pm 4.1$ & $86.44 \pm 2.7$ \\
\hline V & $13.20 \pm 3.4$ & $30.79 \pm 7.9$ & $34.48 \pm 3.3$ & $57.64 \pm 2.6$ & $63.25 \pm 2.2$ & $66.12 \pm 2.8$ & $87.12 \pm 1.8$ \\
\hline VI & $14.87 \pm 3.2$ & $34.68 \pm 8.8$ & $35.21 \pm 1.9$ & $58.82 \pm 1.3$ & $63.42 \pm 1.2$ & $66.65 \pm 1.2$ & $86.50 \pm 1.6$ \\
\hline
\end{tabular}

Table 6: Effect of Thymoquinone Mean Percentile In Paw Edema Volumes In Male Rats Induced By Adjuvant Freund's Complete Adjuvent (FCA)

\begin{tabular}{|c|c|c|c|c|c|c|c|c|}
\hline \multirow{2}{*}{$\begin{array}{c}\text { Dose (mg/kg } \\
\text { body weight) }\end{array}$} & \multicolumn{9}{|c|}{ Days } \\
\cline { 2 - 9 } & $\mathbf{2 2}$ & $\mathbf{2 3}$ & $\mathbf{2 5}$ & $\mathbf{3 0}$ & $\mathbf{3 5}$ & $\mathbf{4 2}$ & $\mathbf{4 8}$ & $\mathbf{5 6}$ \\
\hline \multirow{2}{*}{ Arthritis Control } & 86.46 & 86.41 & 86.16 & 83.85 & 81.46 & 65.65 & 58.81 & 58.34 \\
& \pm 1.9 & \pm 2.1 & \pm 1.8 & \pm 1.5 & \pm 0.83 & \pm 3.3 & \pm 1.56 & \pm 1.2 \\
\hline \multirow{2}{*}{ Vehicle Control } & 85.67 & 85.65 & 81.96 & 70.56 & 64.93 & 61.34 & 58.90 & 55.30 \\
& \pm 2.1 & \pm 1.8 & \pm 1.3 & \pm 1.5 & \pm 1.9 & \pm 1.2 & \pm 3.8 & \pm 4.9 \\
\hline \multirow{2}{*}{ thymoquinone } & 84.43 & 83.94 & 83.42 & 65.96 & 65.04 & 40.81 & 40.44 & 38.65 \\
& \pm 2.9 & \pm 2.9 & \pm 3.2 & \pm 15.6 & \pm 3.3 & \pm 5.4 & \pm 5.1 & \pm 4.8 \\
\hline \multirow{2}{*}{ diferuloylmethane } & 87.24 & 86.92 & 86.34 & 70.56 & 66.74 & 43.11 & 33.47 & 31.32 \\
& \pm 2.8 & \pm 2.6 & \pm 2.1 & \pm 2.7 & \pm 3.3 & \pm 4.1 & \pm 33.7 & \pm 1.4 \\
\hline thymoquinone + & 86.80 & 86.46 & 86.13 & 56.80 & 44.32 & 66.66 & 35.68 & 30.18 \\
diferuloylmethane & \pm 2.1 & \pm 2.4 & \pm 2.3 & \pm 2.7 & \pm 3.2 & \pm 2.8 & \pm 2.5 & \pm 2.1 \\
\hline \multirow{2}{*}{ Standard Drug } & 86.43 & 85.31 & 84.33 & 72.08 & 63.04 & 39.36 & 37.04 & 26.42 \\
& \pm 1.4 & \pm 1.7 & \pm 1.7 & \pm 0.2 & \pm 1.3 & \pm 2.6 & \pm 2.1 & \pm 2.0 \\
\hline
\end{tabular}

Value are expressed as a mean \pm S.E.M $(\mathrm{p}<0.05)$ as compared to control 
Table 6 (continue): Effect of Thymoquinone Mean Percentile In Paw Edema Volumes In Female Rats Induced By Adjuvant Freund's Complete Adjuvant (FCA)

\begin{tabular}{|c|c|c|c|c|c|c|c|c|}
\hline \multirow{2}{*}{$\begin{array}{c}\text { Dose }(\mathrm{mg} / \mathrm{kg} \text { body } \\
\text { weight) }\end{array}$} & \multicolumn{8}{|c|}{ Days } \\
\hline & 22 & 23 & 25 & 30 & 35 & 42 & 48 & 56 \\
\hline Arthritis Control & $86.41 \pm 2.0$ & $85.35 \pm 1.8$ & $83.31 \pm 2.6$ & $82.19 \pm 0.2$ & $75.45 \pm 5.3$ & $63.86 \pm 0.6$ & $58.8 \pm 1.5$ & $58.44 \pm 1.2$ \\
\hline Vehicle Control & $85.57 \pm 2.0$ & $85.44 \pm 1.4$ & $82.44 \pm 2.2$ & $81.09 \pm 1.5$ & $78.50 \pm 2.6$ & $64.14 \pm 1.2$ & $44.28 \pm 0.6$ & $55.30 \pm 4.9$ \\
\hline thymoquinone & $84.43 \pm 2.8$ & $83.76 \pm 2.6$ & $84.32 \pm 3.2$ & $70.58 \pm 2.6$ & $64.95 \pm 3.2$ & $42.23 \pm 5.9$ & $40.45 \pm 5.1$ & $38.85 \pm 4.8$ \\
\hline diferuloylmethane & $86.93 \pm 2.7$ & $86.35 \pm 2.6$ & $84.17 \pm 2.2$ & $72.65 \pm 1.9$ & $65.05 \pm 1.5$ & $43.31 \pm 3.8$ & $39.27 \pm 4.8$ & $36.72 \pm 5.2$ \\
\hline $\begin{array}{l}\text { thymoquinone }+ \\
\text { diferuloylmethane }\end{array}$ & $86.46 \pm 2.1$ & $85.27 \pm 1.9$ & $83.55 \pm 2.0$ & $71.83 \pm 1.7$ & $44.54 \pm 2.6$ & $40.86 \pm 5.3$ & $37.48 \pm 3.8$ & $29.68 \pm 1.2$ \\
\hline Standard Drug & $86.42 \pm 1.3$ & $84.95 \pm 1.4$ & $84.46 \pm 1.3$ & $72.26 \pm 0.1$ & $43.07 \pm 1.5$ & $40.73 \pm 2.8$ & $37.17 \pm 2.1$ & $25.32 \pm 2.8$ \\
\hline
\end{tabular}

Value are expressed as a mean \pm S.E.M $(\mathrm{p}<0.001)$ as compared to control

\section{CONCLUSION}

The result presently conducted study revealed that daily orally administration thymoquinone + diferuloylmethane found to exhibit significant antiinflammatory and the potent anti arthritic activity by significantly $(\mathrm{p}<0.05)$ altering the pathogenesis during arthritis without exerting any side effect during the repeated treatment and proved itself to be the traditionally used and recommended by the practitioner best for the treatment for arthritis when compare to allopathic steroids drug.

\section{ACKNOWLEDGEMENT}

The author are grateful to the Lifescience Intelligentsia Foundation (LIFE) personals for the keen interest and constant encouragement and for the technical assistant Mr. Shadab Alam and Mr. Saleem Ahmed (Department Toxicology) LIFE.

\section{REFERENCES}

Adjuvant Induced Arthritis In Rats. Recent Research in Science and Technology 2010, 2(3): 71-75.

El-Dakhakhany M., 1963. Studies on the Egyptian Nigella sativa L: Some pharmacological properties of its seed's active principle in comparison to its dihydro-compound and its polymer. Arzneim Forsch Drug Res., 15:1227-9.

Elvin-Lewis M., 2001. Should we be concerned about herbal remedies? J. Ethnopharmacol, 75: 141-164.
Ogbonnia S., Adekunle A., Bosa M.K. and Enwuru V.N., 2008. Evaluation of acute and subacute toxicity of Alstonia congensis Engler (Apocynaceae) bark and Xylopia aethiopica (Dunal) A. Rich (Annonaceae) fruits mixtures used in the treatment of diabetes. African J. Biotech, 7(6): 701 -705.

Filippo D’Antuono L., Moretti A. and Lovato A.F.S., 2002. Seed yield, yield components, oil content and essential oil content and composition of Nigella sativa L. and Nigella damascena L. Indust Crops Prod., 15: 59-69.

Ibrahim T., Ali D., Levent E., Mustafa Budancamanak and Demirel A., 2007. Effects of Thymoquinone (Volatile Oil of Black Cumin) on Rheumatoid Arthritis in Rat Models. Phytother. Res., 21, 895897.

Mehtab A., 2013. Subacute 28 days repeated toxicity assessment of thymoquinone (volatile oil of black seed) in wistar rats.

Rassol M. and Sabina E.P., 2007. Anti-inflammatory Effect of the Indian Ayurvedic Herbal Formulation Triphala on Adjuvant-induced Arthritis in Rats. Phytother. Res., 21: 889-894.

Burits M. and Bucar F., 2000. Antioxidant activity of Nigella sativa essential oil. Phytother Res., 14(5): 323-328.

Nickavar B., Mojab F., Javidnia K. and Amoli M.A., 2003. Chemical composition of the fixed and volatile 
ALAM ET AL.: ANTI-INFLAMMATORY EFFECT AND TOXICOLOGICAL QALIFICATION OF COMBINED...

oils of Nigella sativa L. from Iran. Z Naturforsch, 58(9-10): 629-631.

Riaz M., Syed M. and Chaudhary F.M., 1996. Chemistry of themedicinal plants of the genus Nigella. Hamdard Medicus, 39: 40-4.
Siddiqui A.A. and Sharma P.K.R., 1996. Clinical importance of Nigella sativa L. A review. Hamdard Medicus, 39: 38-42.

Siavash P. and Fatehi M., 2003. Effects of Thymoquinone, the Major Constituent of Nigella sativa Seeds, on the Contractile Responses of Rat vas Deferens. Pharmaceutical Biology, 41(8): 616-621. 